\title{
Coronavirus and other pathogens: Reflecting on the relationship between health psychology and infectious disease
}

I think it is fair to say that the broad focus of health psychology in the UK is on noncommunicable disease and lifestyle-related health behaviours. I would like to suggest that perhaps the discipline could devote a little more time to infectious diseases, and not just because we find ourselves in the midst of the coronavirus pandemic. In this reflective piece, I outline some of the assumptions that I held, that I sought to challenge in Stage 1 students, and that I suspect are relatively implicit across health psychology as a discipline.

\section{Reflecting on our teaching practice: is there a gap in health psychology training?}

During my stage 2 training, as part of my Teaching and Training Competence, I had the opportunity to lecture stage 1 students on an MSc in Health Psychology, and I wanted to choose topics that I felt competent enough to take my first lecturing steps with. My PhD research was about antimicrobial resistance and antimicrobial prescribing in veterinary medicine. I was spending a lot of time reading about prevention and treatment of infectious diseases and I realised that this was not just an issue of prescribing decisions. I also took six months out of my PhD to complete a placement at Public Health England, during which I spent a lot of time reflecting on health inequalities and how we might intervene to reduce them.

Given these experiences, I chose the topics of 'tuberculosis', 'infectious disease', and 'health inequalities' as the focus of my guest lectures to the stage 1 students. As health psychologists, we spend a lot of our time thinking about non-communicable disease and promoting healthier lifestyles; important work, of course, given the huge burden of disease associated with modifiable lifestyle behaviours and long-term conditions. When reflecting on my own stage 1 learning, however, I realised that with the exception of HIV/AIDS, I couldn't recall any other discussions about infectious disease, or even other sexually transmitted infections, such as gonorrhoea or chlamydia (both of which can lead to longterm complications if untreated). During my lectures, I therefore wanted to prompt students to pause and reflect on this potential gap in our discipline, and for them to think critically about whether infectious disease is a potentially overlooked topic in health psychology.

I'm sure within the context of the current Covid-19 crisis, many of you will have recognised there is plenty of psychology in dealing with and coping with an infectious disease outbreak. Nonetheless, I would still argue that there is a gap within the discipline that there has been a lack of focus on infectious disease within health psychology. The current pandemic is likely to shift that emphasis; this is welcome of course, but I would also like to highlight that the relevance of health psychology to infectious disease is not just in relation to Covid-19. While the current pandemic reminds us of the truly global nature of infectious disease, it is not the only widespread infectious killer; in 2016, three of the top ten causes of death were related to infectious disease, including influenza, diarrhoeal disease, and tuberculosis (World Health Organisation (WHO), 2018). So, as well as prompting students to pause and reflect, l'd also like you to prompt you, as teachers, researchers, and practitioners, to pause and reflect on three assumptions that I sought to challenge in those lectures. 
Assumption 1: Infectious disease is just about pathogens and microbiology, and needs biomedical solutions - surely psychology can't contribute here?

This was a common assumption I faced during my PhD when I attended microbiology or veterinary conferences to talk about the psychology of antibiotic prescribing decisions by vets and doctors. Fortunately, people were very receptive to the relevance of health psychology, once I'd explained what it was!

Psychology has a lot of influence on the spread or otherwise of infectious disease, and the success of infection prevention and control strategies depends heavily upon behaviour. How well do people perform hand hygiene? How well do people use bed nets to prevent mosquito bites? How well do clinicians achieve getting into and out of personal protective equipment? During the 2014-2016 outbreak of Ebola in West Africa, cultural norms surrounding burial and funeral practices were a factor in the spread of the virus, and many people did not attend healthcare facilities for early treatment (for Ebola and other conditions), due to fears about catching Ebola at such facilities. The Covid-19 pandemic is highlighting to all of us how everyday interactions and behaviours, such as hugging, handshaking, and touching faces and surfaces, are all potential transmission points for many pathogens.

Even where treatment is available, the use of antimicrobial therapies is not based solely on clinical factors. Doctors' and vets' prescribing decisions are partly influenced by emotions, especially fear, and also by local prescribing norms within a practice or hospital ward. Adherence to antimicrobials by patients is also an issue, with many ceasing treatment courses early or taking medication incorrectly, which can lead to drug-resistant infections. There can also be stigma associated with infectious diseases, which can prevent people seeking treatment or result in ostracization from the community. Psychology can even influence our susceptibility to disease, with one series of studies highlighting that psychological stress and negative life events are associated with increased infection rates following exposure to a common cold virus (Cohen \& Janicki, 2011).

Assumption 2: Infectious disease isn't really a priority in HICs - we have vaccines, good sanitation, effective public health programmes, and robust health systems. We have other things to worry about in the UK, surely?

Infectious disease is an issue for high-income countries (HICs), even when we set aside how effectively the current Covid-19 pandemic challenges the above notions. First, HICs have infectious disease, even if it seems less salient to us. Second, we live in a globalised and highly interconnected world where outbreaks can quickly spread. Third, we are all part of humanity and should be just as concerned with addressing global, as well as local, inequalities.

Yes, in the UK and other HICs, the burden of disease from non-communicable diseases is much greater than from infectious disease. But infectious disease is still relevant in HICs. Consider the recent increases in measles cases across Europe associated with falls in the MMR vaccination rate; health psychology can surely contribute to help strengthen public trust in vaccines and counter misinformation. In 2018, there were an estimated 2.4 
million cases of food poisoning in the UK; maybe we need more effective food hygiene messaging targeting the home, or those working in food outlets? And what about the trend for 'raw' milk? Pasteurisation kills human pathogens such as Salmonella, Campylobacter, and Escherichia coli, and is generally considered a very effective public health intervention, so why do some people believe that pasteurisation can do more harm than good?

Furthermore, if as a discipline we care about addressing health inequalities, then we need to work more with infectious disease. In the UK, tuberculosis (TB) is a relatively rare condition in the general population, but TB infection is closely associated with social and economic inequalities; TB is more prevalent in disadvantaged and marginalised groups, such as the prison population, the homeless, and those with histories of substance abuse. Globally, 95\% of deaths from TB are in low- and middle-income countries (LMICs). Indeed, the proportion of the disease burden attributable to infectious disease in general is much greater in LMICs than in HICs, and the social determinants of health (such as poor housing, lack of access to healthcare, and economic hardship) are all related to patterns of infectious disease.

And let's not forget the neglected tropical diseases (NTD). The WHO states there is "overwhelming evidence to show that the burden caused by many of the 17 diseases that affect more than one billion people worldwide can be effectively controlled and, in many cases, eliminated or even eradicated" (WHO, 2012; my emphasis). Most NTDs are preventable, but they can cause irreversible problems and the burdens on people and economies are huge. If, as a discipline, we are not paying enough attention to infections, are we also contributing to these local and global health inequalities?

\section{Assumption 3: Infections are short-term illnesses, with acute effects, and relatively quick treatment. Once recovered, people go back to life as normal. Surely the impact is minimal?}

For many of us in the UK, at least prior to 2020, we might tend to think of infectious disease as being a transient issue; colds, urinary tract infections, eye infections - these are generally short-lived, easily treated, and rarely have lasting impacts. In many cases, however, infectious disease is neither short-lived nor without long-term impacts.

Not all infections are cleared quickly. Treatment for TB requires a six-month course of combination antibiotics, and if you are unlucky enough to have a drug-resistant strain of $T B$, then you are looking at a minimum of eighteen months on antibiotics, with a reasonable expectation of some pretty rough side effects. Some infections cannot be cured; we can only offer supportive therapies during an illness and hope the immune systems clears the infection, or in the case of HIV, offer lifelong treatment. Even for infections that are shortlived, there can still be long-term effects; for example, measles can cause meningitis with the risk of long-lasting brain injury, shingles around the eyes can lead to vision loss, malaria can cause seizures and lead to kidney failure, and polio can cause paralysis and muscle weakness. And of course, people can die from infectious diseases.

Aside from the physical impacts, there are also psychological, social, and economic impacts on patients and those around them. People have to adapt and learn to cope with 
lifelong disabilities, children may miss significant amounts of schooling, adults may have to leave the labour market, and families experience distress and grief when their loved ones are seriously ill or have died. In HICs, the impacts of infectious disease may be lessened by access to good healthcare, but it is a mistake to equate infectious disease with minimal impacts.

\title{
Challenging our assumptions
}

I know there are health psychologists out there who are already working in infectious disease. In the current context, many more of you are likely to be looking at ways to contribute by understanding, for example, how people cope with lockdown, the effects of losing someone to Covid-19, or of surviving it, and many other important questions. All of these efforts are much needed.

I am not, however, suggesting that we all start working in infectious disease, just as we are not all working in long-term conditions, physical activity, or stress. Preventing noncommunicable disease is still important, and that work remains just as valuable. What I am suggesting, is that we each consider how we react to the current pandemic in terms of our teaching material (and perhaps our future grant applications). I wonder - how many of the above assumptions do we and our students implicitly endorse? I know I did before I commenced my PhD and Stage 2 training. We encourage our students to be critical, but shouldn't we also be critical of ourselves, our curricula, and our discipline?!

From a teaching perspective, infectious disease can also be a useful vehicle for prompting broader discussions with students, such as some of the moral and ethical challenges associated with limiting the burden of disease or allocating access to limited resources. How do we set our research and funding priorities? When should we focus on the individual versus the population? How do healthcare workers balance such challenges, in times of crisis and in 'normal' times? If and when a safe and effective vaccine is developed for Covid-19, who should get early access? In the UK, the calls are for health and social care workers to be vaccinated first - but should they be? Perhaps the first batch should go to healthcare workers in poorer countries, where for many people there is limited scope for meaningful hand hygiene or social distancing, and where, if you get very sick, the chance of you accessing a ventilator is slim to none. I don't profess to know the answers to these difficult questions, but health psychologists should certainly be contributing to these debates.

Now is clearly not the time for drastic change; there's enough of that already in the world, and we need to be focusing our energy on caring for ourselves and our loved ones and maintaining whatever level of 'normal' we can manage for those we work with. In the future, however, when there is time to reflect, I would urge you to consider whether infectious disease could be better reflected in our Stage 1 courses, and perhaps in the discipline itself. In the meantime, stay safe, and wash your hands. Properly.

\author{
Author: Dr Sarah E. Golding \\ Postdoctoral Research Assistant in Health Psychology \\ University of Winchester
}


golding.sarah@icloud.com

Twitter = @SarahEGolding

Acknowledgement: Many thanks to Professor David Sheffield for reminding me about the work of the Common Cold Project.

\section{References:}

Cohen, S., \& Janicki, D. (2011). The Common Cold Project - Carnegie Mellon University. Retrieved from https://www.cmu.edu/common-cold-project/

WHO. (2012). Accelerating work to overcome the global impact of neglected tropical diseases: A roadmap for implementation. Executive Summary. Retrieved from http://apps.who.int/iris/bitstream/handle/10665/70809/WHO_HTM_NTD_2012.1_eng .pdf? sequence $=1$ \&isAllowed $=y$

WHO. (2018). The top 10 causes of death. Retrieved from https://www.who.int/en/newsroom/fact-sheets/detail/the-top-10-causes-of-death 